\title{
Performance engineering, PSEs and the GRID
}

\author{
Tony Hey and Juri Papay ${ }^{\mathrm{b}}$ \\ a Director UK e-Science Programme EPSRC, Polaris House, North Star Avenue Swindon SN2 1ET, UK \\ E-mail:Tony.Hey@epsrc.ac.uk \\ ${ }^{\mathrm{b}}$ Department of Electronics and Computer Science, University of Southampton, Southampton SO17 1BJ, UK \\ E-mail:jp@ecs.soton.ac.uk
}

\begin{abstract}
Performance Engineering is concerned with the reliable prediction and estimation of the performance of scientific and engineering applications on a variety of parallel and distributed hardware. This paper reviews the present state of the art in 'Performance Engineering' for both parallel computing and meta-computing environments and attempts to look forward to the application of these techniques in the wider context of Problem Solving Environments and the Grid. The paper compares various techniques such as benchmarking, performance measurements, analytical modelling and simulation, and highlights the lessons learned in the related projects. The paper concludes with a discussion of the challenges of extending such methodologies to computational Grid environments.
\end{abstract}

\section{Introduction}

Performance has been a central issue in computing since the earliest days [3]:

'As soon as the Analytical Engine exists, it will necessarily guide the future course of science. Whenever any result is sought by its aid, the question will then arise - by what course of calculation can these results be arrived at by machine in the shortest time?'

Performance engineering may be defined as a systematic approach in which components of both the application and computer system are modelled and validated. Although performance is probably one of the most frequently used words in the vocabulary of computing, paradoxically it is evident that there is a substantial "knowledge gap" between the software development process and actual performance estimation and optimisation. It is still often the case that programmers and software system designers have insufficient knowledge of the performance implications of their design choices. Indeed, it is clear that systematic performance engineering is not yet an integral part of the software development process and that performance issues often arise very late in the process. As a result it is not surprising that performance problems are a frequent cause of failure of large software development projects.
One possible reason why performance issues do not feature explicitly in current software methodologies is Moore's Law. Up to now, the exponential growth in microprocessor performance has usually enabled users to avoid hitting any serious 'performance-wall'. However, in the relatively near future, it is likely that growth in processor performance will slow down and begin to deviate from Moore's Law. Software developers will then be forced to pay more attention to the efficient use of the available silicon real-estate. Furthermore, if the Grid becomes a reality and computer resource 'marketplaces' begin to emerge, software performance on different hardware platforms will be directly related to real costs. In such a 'computational economy', it is clear that performance engineering and reliable performance estimation will play a pivotal role in the establishment of realistic 'performance contracts'. A performance contract is the product of the negotiating process between the suppliers and customers of computing resources. It contains information about the resource demand of applications and available computing capacity. At present this feature is not available, this is mainly due to the lack of reliable performance estimation techniques. The Grid is assumed to be 'an infrastructure that enables flexible, secure, coordinated resource sharing among dynamic collections of individuals, institutions and resources' [13]. The resources accessible 
via the Grid include computational systems, data storage and specialized facilities and are thus a richer set of 'informational utilities' than the Web. In this context it is helpful to consider the Grid as providing the global middleware infrastructure that will enable the establishment of transient "virtual organizations" on a transparent and routine basis.

There will be many different types of applications for the Grid and in many cases it is likely that Problem Solving Environments (PSEs) generalized to the Grid will play an important role. A PSE is an applicationspecific environment that provides the user with support for all stages of the problem solving process from program design and development to compilation and performance optimisation. Such an environment also provides access to libraries and integrated toolsets, as well as support for visualization and collaboration. It may also implement some form of automated workflow management. Performance engineering - including estimation, monitoring and measurement - will be an integral component of any Grid PSE since reliable models of performance prediction will be required for any realistic Grid scheduling and accounting packages.

The paper is organized as follows. Section 2 reviews several different approaches that have been attempted for performance engineering and gives a short account of some performance benchmarking, monitoring and simulation techniques. Section 3 takes a brief look at Problem Solving Environments in the context of performance and presents a short account of two recent UK-based PSE projects. The next section outlines some of the challenges represented by the Grid for the performance evaluation community and reviews some EU experiments on Europe-wide meta-computing. Finally we offer some conclusions and challenges for the performance engineering community.

\section{Performance engineering approaches}

\subsection{Benchmarks}

The goal of benchmarking is to understand and predict the key parameters that determine the performance of computing platforms and full scale applications. There have been numerous benchmarking efforts undertaken in the past but no general agreement on how to conduct the measurements and how to interpret the results. Examples of benchmarking efforts include the Livermore Loops [38], the NAS Kernels [4] and the Parkbench initiative [16]. It is worthwhile for us to summarize the objectives and achievements of Parkbench.

The main objectives of the ParkBench initiative were:

1) To establish a comprehensive set of parallel benchmarks that is generally accepted by both users and vendors of parallel systems.

2) To provide a focus for parallel benchmark activities and avoid unnecessary duplication of effort and proliferation of benchmarks.

3) To set standards for benchmarking methodology and result-reporting with establish a database/ repository for both benchmarks and the results.

4) To make parallel and sequential versions of the benchmarks and results freely available in the public domain.

As a result of this effort a benchmark suite was developed which contains sequential and message passing versions of the following codes:

- 5 Low Level Communication codes

- 5 Low Level Sequential codes

- 5 Parallel Linear Algebra Kernels

- 2 NAS Parallel Benchmark Kernels

- 3 NAS Compact Application codes

- ORNL Shallow Water Model Application code

Open-MP versions of some of these codes are now available [18]. By providing three tiers of benchmark complexity - low-level, kernel and compact application - it was hoped that the performance of real applications could be understood. The low-level codes provide basic machine parameters, the kernels provide information about compute intensive algorithms and the compact applications add the complexities of start-up, I/O and so on. In the event, Parkbench was only partially successful: lack of dedicated funding for such a benchmark evaluation programme prevented its full exploration and realization. Nonetheless, there were significant achievements - a serious programming methodology was defined, parallel versions of the NAS Parallel Benchmarks were made available in the public domain and a repository for results with a graphical interface established. In addition, an electronic journal for the rapid publication of performance results was established [19] and this is now established as a special section of the journal Concurrency and Computation: Practice and Experience.

Two examples will illustrate the type of data that was made available by these benchmarks. Figure 1 shows the performance of the parallel LU kernel benchmark 


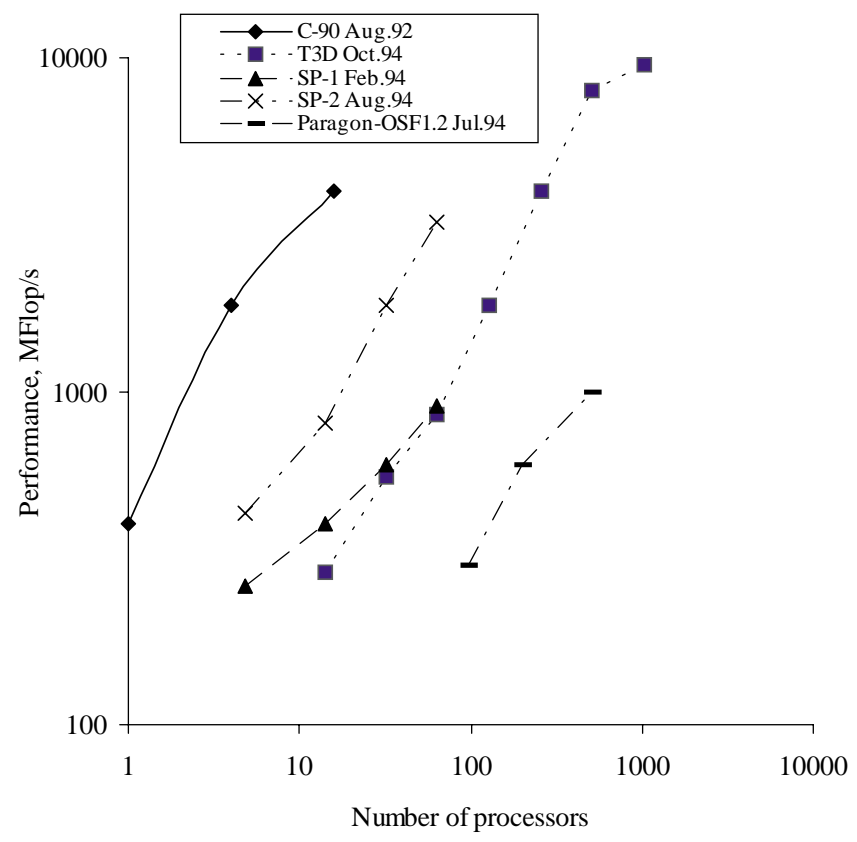

Fig. 1. Performance of vector vs. distributed memory machines on LU kernel.

on distributed memory (DM) machines. The data is from 1994 and shows for perhaps the first time the performance of a parallel DM system outperforming the largest vector supercomputer of the time. Figure 2 shows results of the low-level communication benchmark COMMS1 for the Intel Delta and the Intel iPSC/860 systems.

The main concern of performance engineering is to develop techniques which enable to predict the performance and resource requirements of applications, in this sense benchmarking has little to offer. The results of benchmarks are usually expressed by a single number which is sufficient for comparing and rating various computers, however this number provides little information about the key parameters governing the resource requirements of real applications.

\subsection{Performance measurements}

Performance measurements are based on event profiling and tracing. These techniques assume an event model of program execution. During program execution, profiling tools accumulate summary data for significant events such as function calls, cache misses, communications etc. Typically, this approach has a low overhead since such profiling is usually implemented by simple event counters. Using this method users can obtain statistical information on the percentage of time spent by their application program in performing vari- ous functions and can use this information to identify potential problem areas.

Trace tools on the other hand, provide the user with much more detailed information on the sequence of events as they happen during program execution. Trace files record time ordered events and can constitute a large volume of data. The information recorded in a trace can represent various levels of abstraction. In the case of parallel platforms, the recorded traces for the different nodes need to be collected, sorted according to time stamps and merged into a global event trace. Although trace tools provide more detailed information about the parallel program execution than the profiling tools, there can be a significant overhead for trace generation. Furthermore, the large volumes of trace data generated can be overwhelming.

There are numerous commercial and academic tracing and profiling tools available. Examples include Apprentice [8], gprof, Vampir [41] and Paradyne [5]. Apprentice is a product of Cray Research which uses source code instrumentation through compiler switches to provide statistics on the level of functions and basic blocks. The advantage of source code instrumentation is that the results of monitoring can be easily interpreted in terms of programming language statements and give very direct feedback to the programmer. A problem with this approach is that the libraries cannot generally be monitored at the same level of detail since they are usually only available in binary format. Unlike the 


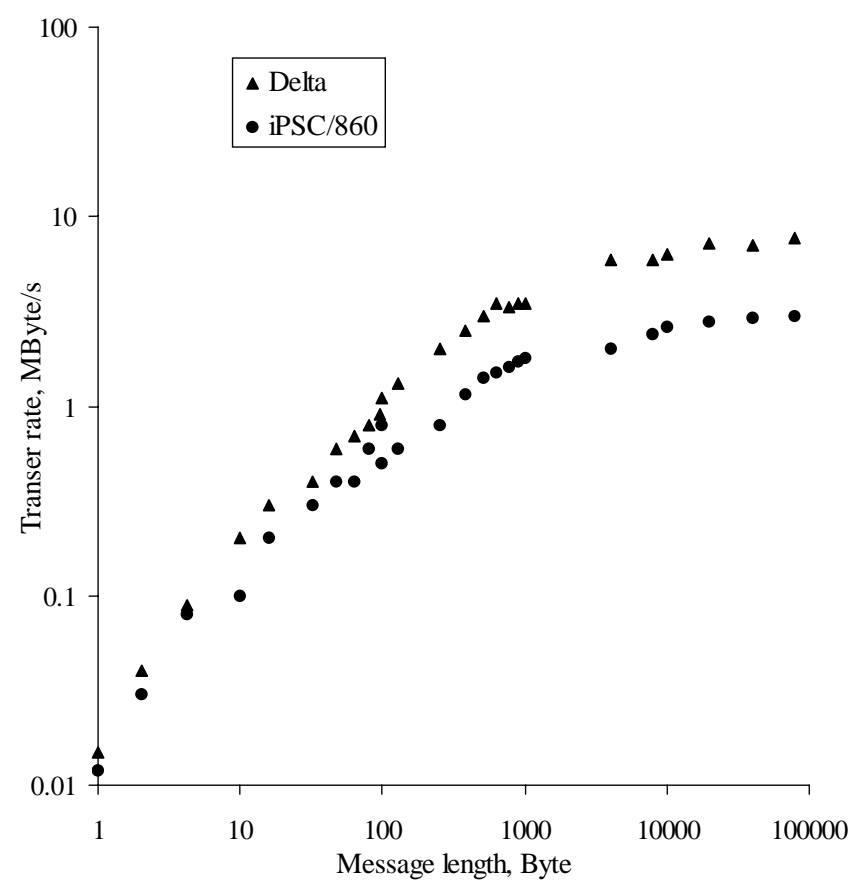

Fig. 2. Benchmarking of communication latency.

Cray product, Vampir is a commercial graphical event trace browser from Pallas that is available on many different platforms. Vampir also provides visualization and statistical analysis of trace files.

Measurement and profiling tools have achieved a high level of maturity, however the usage of these tools for reliable performance estimation is still an open issue. These tools can generate a large volume of detailed data, but in order to gain some understanding of the application's runtime behaviour this data needs to processed and interpreted. The interpretation is not a simple and straightforward process, it requires user intervention and considerable knowledge of the problem domain. An important aspect of these tools is the level of intrusion which affects the accuracy of measurements and can even alter the behaviour of the system, this is often not mentioned or not quantified. The amount of data that is collected during the measurement is related to the level of intrusion, therefore it is vital to find the right balance between the volume of data and the acceptable level of intrusion.

\subsection{Analytical models}

Historically, Hockney's $n_{1 / 2}$ and $r_{\infty}$ 'pipeline' model provided a useful abstraction of Vector Supercomputer architecture [15]. This pipeline model has been extended to characterize communication performance in parallel DM message-passing systems. In this case the pipeline parameters captured the communication latency and asymptotic communication bandwidth. Typically, the nodes of such systems are scalar processors but it is also possible to use Hockney-style pipeline parameters to provide a simple characterization of the memory hierarchy of the node. These are basic hardware parameters. It is also possible to characterize the 'computational intensity' of an application in terms of the ratio of the number of arithmetic operations performed per off-chip memory access. The parallel program is represented as an alternation of nonoverlapping computation and communication stages. The application program is described in terms of the number of scalar floating point operations, the amount of data transferred and the number of messages. The output of the model is assumed to be the sum of processing and communication times. The model assumes perfect load balance and the timing formula derived for a single processor is used for the performance characterisation of the whole parallel program. A weakness of this model is that it is only valid for the performance analysis of parallel algorithms with regular structures and good load balancing.

In recent years, several other cost models have been developed. These include the BSP (Bulk Synchronous 
Parallel) model [46,36,37] and the LogP model [9]. Both these approaches attempt to get beyond the usual (unrealistic) assumptions made in 'classical' PRAM complexity analysis. In particular an attempt is made to take into account the limitations of real systems such as the network bandwidth, and communication and synchronisation overheads. These models aim to provide a machine independent framework for parallel algorithm design and performance prediction. The $\log P$ model characterises the parallel system by the following parameters:

- the upper bound on communication latency from source to target $(L)$,

- the overhead of send and receive $(o)$,

- the minimum time interval between consecutive transmissions or receptions $(g)$,

- the number of processor/memory modules $(P)$.

The model assumes asynchronous execution mechanism, finite network capacity and specifies the work $(W)$ between communications.

The BSP model, on the hand, has an equally simplistic and unrealistic cost model. In fact, the Oxford 'BSP model' bears almost no resemblance to Valiant's actual BSP complexity analysis. In order to prove any useful results, in his original BSP model Valiant requires parallel slackness at the nodes to hide communication delays, two-phase random routing of messages to avoid possible network congestion, and data hashed randomly across the processors with a sufficiently random class of hash functions to avoid memory 'hot spots'. All that is left of Valiant's BSP analysis in the Oxford BSP model is the programming methodology of the bulk synchronous programming style! Both the BSP and LogP models are similar in a sense that they attempt to provide an abstraction of the performance of the communication network and processing nodes using a minimal number of 'average' performance parameters. Details of the network topology and memory hierarchy are ignored. Such models represent, at best, a "back-of-the envelope" approach to performance prediction. Nevertheless, it must be said that in some cases, as the experiments on CM-5 showed, the LogP model was able to provide a close match to actual performance measurements [11]. As we will see below, a similar 'average' speed analysis of the Livermore loops ignoring any effects of the memory hierarchy gives performance results that can be over $100 \%$ under- or over-estimated. Other interesting approaches to performance modelling include Carter's Parallel Memory Hierarchy Model [2] and the Manchester group's Overhead Analysis approach [40,44].
Analytical modeling is in fact complexity analysis, which involves an abstract model of program execution and cost models of computation and communication operations. This technique attempts to combine the parameters of the application and the computer in order to produce a mathematical expression for performance estimation. This approach involves numerous assumptions which approximate the system and application's behaviour. Although these approximations make the performance evaluation analytically tractable, they significantly reduce the accuracy of predictions and limit the applicability of analytical models to certain class of applications or computer architectures.

\subsection{Simulation}

Simulation can provide very detailed information about both the computer system and application program. This information can be at various levels, in terms of hardware architecture ranging from simulation of only the main components of the architecture right down to simulations at the gate level, and on the application side, from programming language statements down to machine code. The simulation model characterizes the system by a number of state variables that are updated as the simulation progresses. Simulation techniques can be classified according to four basic types: instruction driven, trace driven, execution driven and event driven. Instruction driven simulation is based on interpreting instructions of the target machine. This technique gives high accuracy, achieved by step-bystep simulation of each instruction, but requires long simulation times. Full instruction level simulation is too time consuming for practical use on real applications and complex machine architectures.

Trace driven simulation uses records of measurements obtained from the real system or synthetic traces generated by the trace generator. The trace is a sequence of user defined events generated by an instrumented program. This technique can require large amounts of memory and processing time to produce reliable results. The DIMEMAS tool is an example of a trace-driven performance prediction tool for messagepassing parallel programs [34]. This tool uses the Vampir Trace File and scales the CPU time spent in each block of code and the parameters of communication events according to the target machine parameters.

Event driven simulation maintains a global queue of events. The operation cycle consists of event fetching, the simulation step and update of the data structure representing the simulated system [42]. The inputs of 


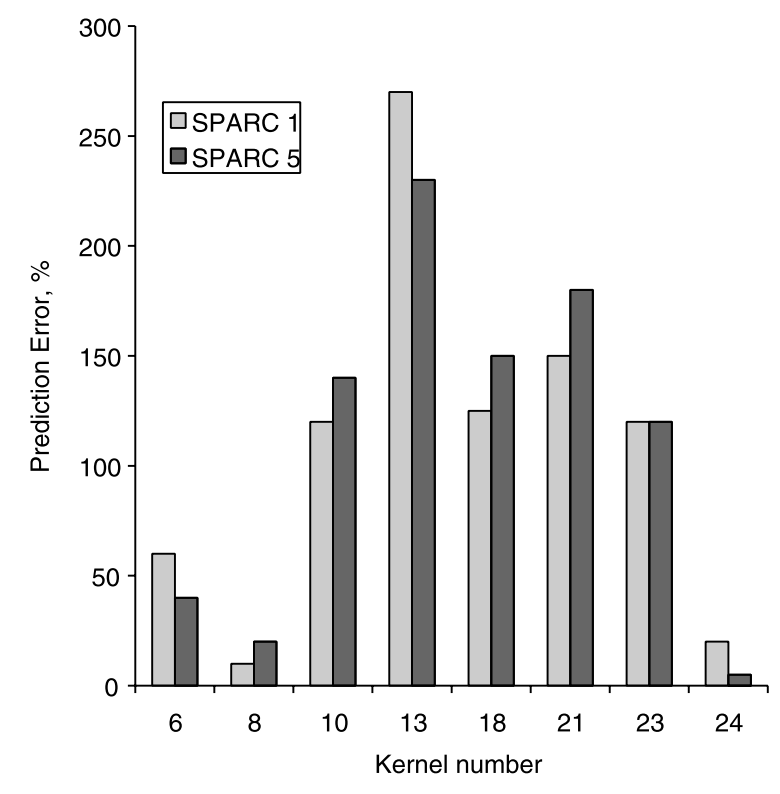

Fig. 3. Prediction error of static statement analysis.

event driven simulation models are probability distributions of response times, request arrivals and delays. The main disadvantages of probabilistic workload models are that they do not directly represent the parameters of specific application programs and the results of the simulation require in-depth statistical processing in order to determine the accuracy of the model.

Finally, execution-driven simulation models interleave the execution of an application with the simulation of the target system. The main advantages of execution driven simulation are the speed and the use of actual programs for the simulation of parallel architectures rather than using distribution or trace driven workloads. That such an execution driven approach is a feasible solution for the simulation of parallel systems has been demonstrated by systems such as the Rice Parallel Processing Testbed (RPPT) [7] and the Wisconsin Wind Tunnel [43]. The disadvantage of this technique is that it is more difficult to implement than the trace driven approach due to the complex interactions between the application program and the simulator.

A key lesson learned from the previous work is that any simulation method must take account of memory hierarchy for realistic performance estimation. Simple static statement analysis of the source code is well known to be unpredictably unreliable as it is presented by Fig. 3, which shows the difference between predicted and actual execution times of Livermore Fortran kernels on SPARC 1 and SPARC 5 workstations.
The PERFORM system developed by Dunlop at Southampton is an execution-driven simulation tool that uses a novel 'Fast Simulation Method' that attempted to improve the accuracy of prediction and overcome some of the problems with a full simulation of the memory hierarchy $[10,14]$. The model uses the "program slicing" technique to isolate the control variables and array indices of the source code, retaining sufficient information to simulate data movement within the memory hierarchy. The sliced program is then augmented with calls to the PERFORM simulator which models the effects of memory hierarchy cache memory, computation and message passing. Fast simulation is achieved by providing feedback between the simulator and source and curtailing loop execution when the cache behaviour of iterations are reliably estimated. The main stages of the Fast Simulation Method used in PERFORM tool are illustrated in Fig. 4.

An example of the accuracy of predictions that can be achieved by the PERFORM tool is shown in Fig. 5 that compares actual and predicted performance on a SPARC system. As can be seen, the predicted lower bound provided by PERFORM captures the detailed cache effects very accurately.

Simulation is a useful technique for the performance evaluation of systems at the design stage of development. Concerns with such simulation-based approaches are the level of detail of the simulation model, accuracy and the simulation time. Simulation models are usually large size programs, their development is expensive and in the case of simulations of the architecture at the instruction level for example, require a long run-time in order to provide meaningful results.

\section{Problem Solving Environments}

A Problem Solving Environment (PSE) is an integrated computing environment which incorporates all stages of the problem solving process, such as problem specification, computation, analysis and optimization. The key issues associated with the architecture design of PSEs are interoperability, modularity and reusability of components. The problem of interoperability of the different software packages stems from the different (and often proprietary) file formats produced by the various components. This is often the case, for example in mechanical engineering where we frequently need to implement data exchange between various CAD packages and CAE tools. Several recent papers [20,21] highlight the need to adopt a universal file format based 


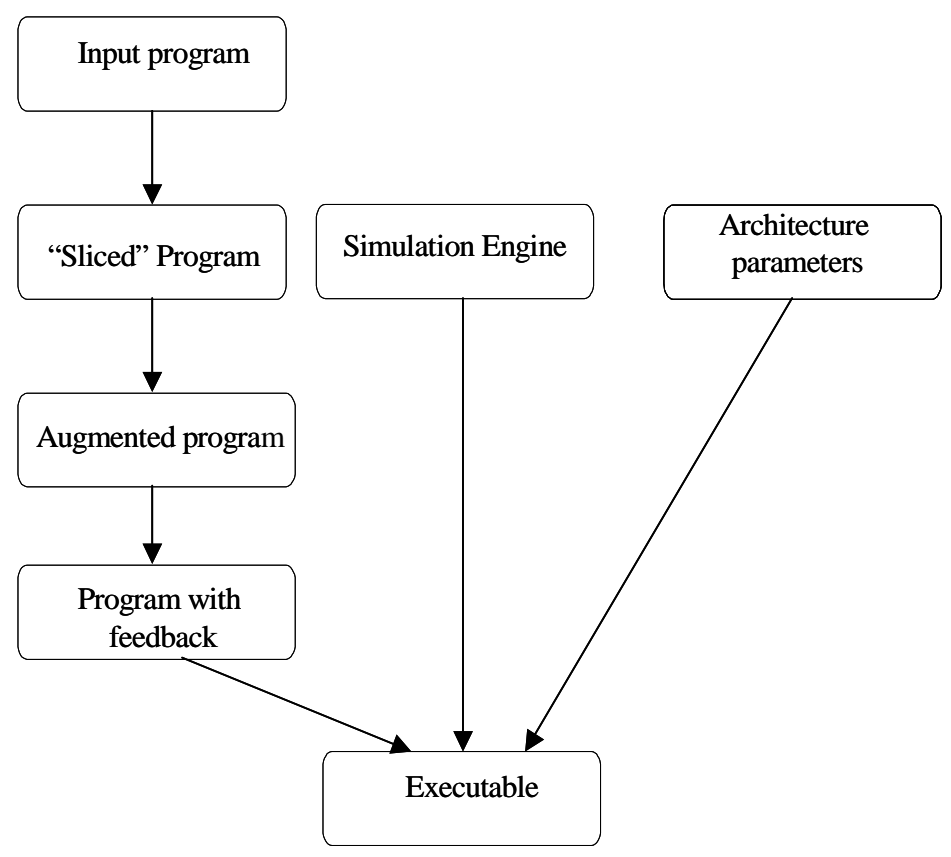

Fig. 4. Key stages of fast simulation method.

on XML that will simplify data exchange and structure specification. Many of these pleas are from users with real industrial applications but vendors of component software packages see little commercial incentive to make their software easily interoperable with packages from other vendors. At present there is no generally accepted methodology for the specification, analysis and design of modular reusable systems.

In recent years there has been a significant progress in the development of middleware technologies that provide support for system integration based on objects. Examples of these technologies include CORBA, DCOM and, in the context of Web Services, the recently proposed SOAP protocol. Although these technologies share many common features there is no universally accepted definition of objects and consequently their claims to provide full interoperability within the same system are somewhat questionable. At present, CORBA is the dominant middleware technology in the PSE world. Key advantages of CORBA are the existence of a single specification document and the participation of more than 800 companies in the consortium. Nevertheless, despite the existence of the IIOP inter-ORB protocol, there is still a problem for applications that attempt to mix two or more of the large number of vendor specific implementations of CORBA. An unwelcome result of this diversity is the problem of interoperability between competing middleware prod-

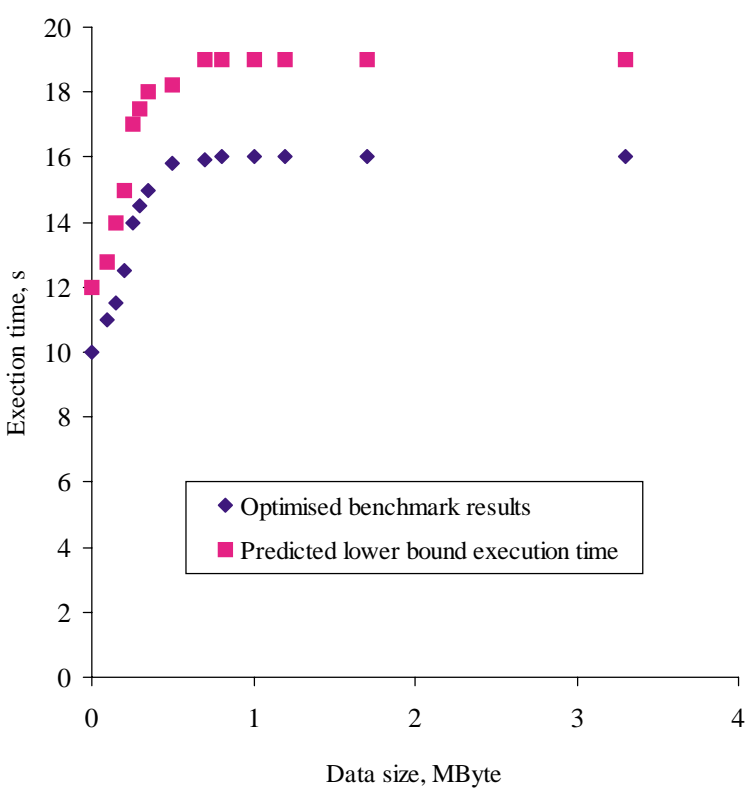

Fig. 5. Predictive power of PERFORM tool.

ucts. It should also be emphasised that programming using any of the CORBA implementations is not trivial and the $\mathrm{C}++$ syntax is rather complex. By contrast, DCOM is Microsoft's answer to CORBA and this has the definite advantage that there is one specification, implementation and one vendor. A limitation is that DCOM runs only on Windows. 


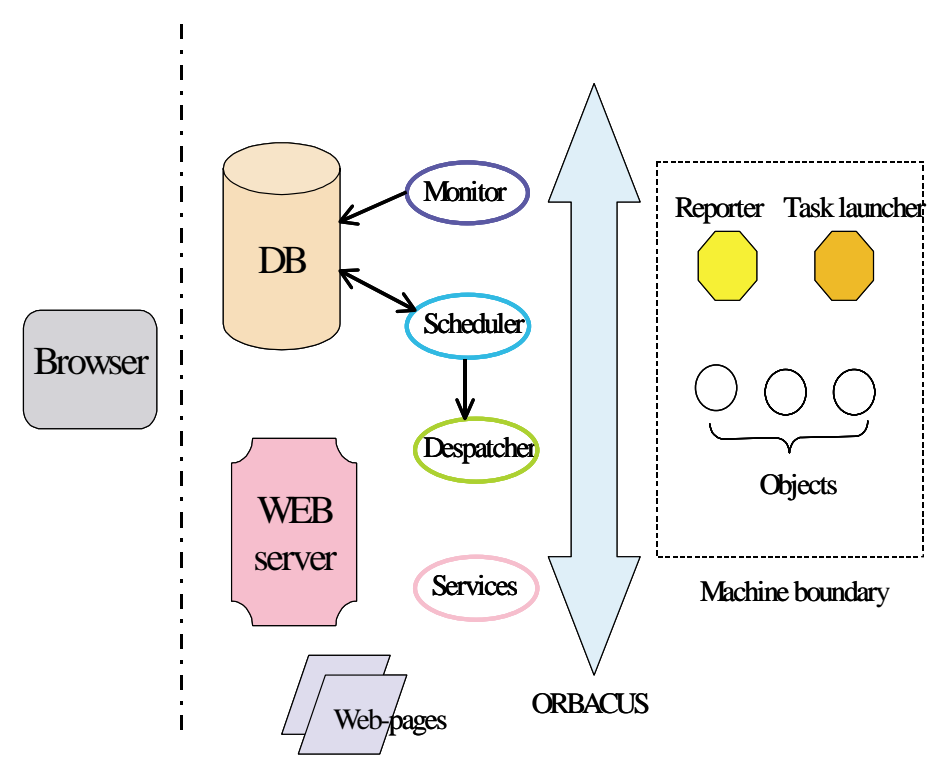

Fig. 6. A PSE architecture built from Cardiff/Southampton PSE components.

Arguably, two of the most successful PSEs are the commercial products Matlab and Mathematica. Both these mature software environments have successfully combined good usability with functionality. Perhaps, strictly speaking, these products do not qualify as genuine PSEs since they both provide rather generic environments for a broad range of application areas rather than an environment targeted at a single application. Nevertheless, these products have a wide user base and demonstrate what is possible in principle though neither have seen the development of a version for parallel systems as a high commercial priority. Apart from these examples, there are many 'research' PSEs either in existence or under development in many universities and research institutes around the world. Examples include GasTurbnLab from Purdue [22], BIOPSE from Utah [23] and Autobench from Stuttgart [24]. Unfortunately, it is not clear whether any of these PSE systems are much used by real users.

As two examples of PSE projects, we shall briefly describe two ongoing UK projects: one a project with considerable direct industrial involvement and the other a purely 'academic' project. These are the Swansea/BAE Systems Project [49] and the Cardiff/Southampton PSE project [47]. The Swansea/BAE Systems project represents a complete industrial environment for multi-disciplinary computational fluid dynamics, electro-magnetics and structural mechanics simulations. This PSE includes geometry builder, mesh repair, unstructured grid generation, grid quality analysis, post-processing and data analysis, execution on remote/parallel platforms, help facilities and application integration. The system is based on CORBA and uses a parallel architecture (VIPar) for image processing. This PSE has been further developed in the CAESAR and JULIUS EU projects. A key problem for implementation of their 'Computational Science Pipeline' is the data transfer between the different components.

The aim of Cardiff/Southampton PSE Project is to leverage modern software technologies such as CORBA, Java and XML and to develop the key modules which can be used for the rapid prototyping of application specific PSE environments [47]. The main components developed in this project are: a Visual Component Composition Environment, an Intelligent Resource Manager, based on the Southampton Intrepid Scheduler [1] and a Software Component Repository. The two applications targeted by this project are Molecular Dynamics and Photonic Crystal Structures simulations. A PSE architecture incorporating the developed components is presented in Fig. 6.

The system is based on the object-web concept where the services are represented as network objects. The problem is formulated as an XML request by the user and the response, also in XML, is produced by the Web server. The user interface is embedded in the browser environment and enables visual programming by allowing "drag-and-drop" of objects in a task-graph design area. The interaction with the user is implemented as a sequence of Web pages. As a commercial middleware ORB, the ORBACUS implementation of CORBA 
was selected which is a mature product and provides numerous services for naming, trading and interface repository.

The main task of the Monitor is to collect and store information about machines and tasks running in the system. The information about machines includes data about the available resources such as memory, processors, disk and load. The task information is a representation of the resources used by the given task such as size of occupied memory, communication and I/O traffic, and disk volume used. The information collected by the Monitor is stored in a database and used by the Scheduler for task allocation and load balancing. The interactions of Monitor with the other components of the system are represented in Fig. 7. On each computer there is an Object Server deployed which instantiates the Reporter object. The Reporter registers with the Name Server, which maintains a list of remote object addresses. The Monitor at regular intervals queries the Reporter objects and updates the Machine and Task tables in the database.

The Scheduler provides task allocation to resources, run-time forecast and dynamic load-balancing. The scheduling is based on machine independent application load models for CPU, memory size, I/O traffic and disk volume. These algebraic expressions are included in the description of each task and represent the task resource requirements. The scheduling algorithm performs the following steps for each task in the task-graph:

- check the availability of licenses, memory, disk

- generate list of candidate machines

- compute time components

- select minimum execution time

- include task-machine binding in the schedule

The three year project is nearing completion and a full evaluation with performance measurements will be available soon. Preliminary indications are that such a component based approach can bring real advantages in terms of software development and deployment. In the final analysis, however, it will be the reaction of users to such an environment that will provide the real measures of success or failure!

On the whole, the lessons learnt from many of these PSE experiments are not very encouraging. There are major problems in automating the data flow between CAD and CAE tools. Furthermore, there is little incentive for vendors of legacy codes to make their product interoperable with tools from other vendors. In addition, present PSEs focus almost entirely on the design and simulation part of the engineering process. There is a real need to incorporate the experimental validation and testing part of the process. Thus a complete PSE would offer support for the recording and analysis of experimental as well as simulation data. Incorporation of databases of experimental measurements and simulation results will allow the development of data mining and knowledge discovery components of the PSE. PSEs have a long way to go to prove their worth in real engineering environments!

PSEs often represent large scale meta-applications which require massive computing resources. In this case the role of performance engineering is to predict the resource requirements of the application, to ensure that there is sufficient computing capacity available and the individual tasks are assigned to the most appropriate computer.

\section{Grids}

\subsection{The Grid as a new paradigm}

The significant investments currently being made in Grid research shows that the governments around the world are taking the development of such infrastructure middleware very seriously. With the recent announcement of IBM's support for the Grid, it is not unreasonable to expect that the Grid will eventually become the key middleware not only for science and engineering but also for industry and commerce.

In the US several agencies are funding major Grid initiatives. Examples include:

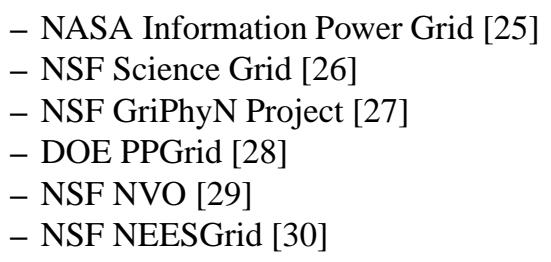

Most of these Grid Infrastructure and Application Projects make use of the Globus Toolkit [12] as the basic platform on which to provide Grid services. In addition, the NSF funded GrADS project [6] identifies many important research issues for Grid computing. Europe has been also active in Grid R\&D. In addition to two initial EU Grid projects, DataGrid [17] and EuroGrid [31], several new EU Grid-centred projects are currently under negotiation. National governments in the EU have also recognised the potential strategic importance of the Grid. For example, under its new 'e- 


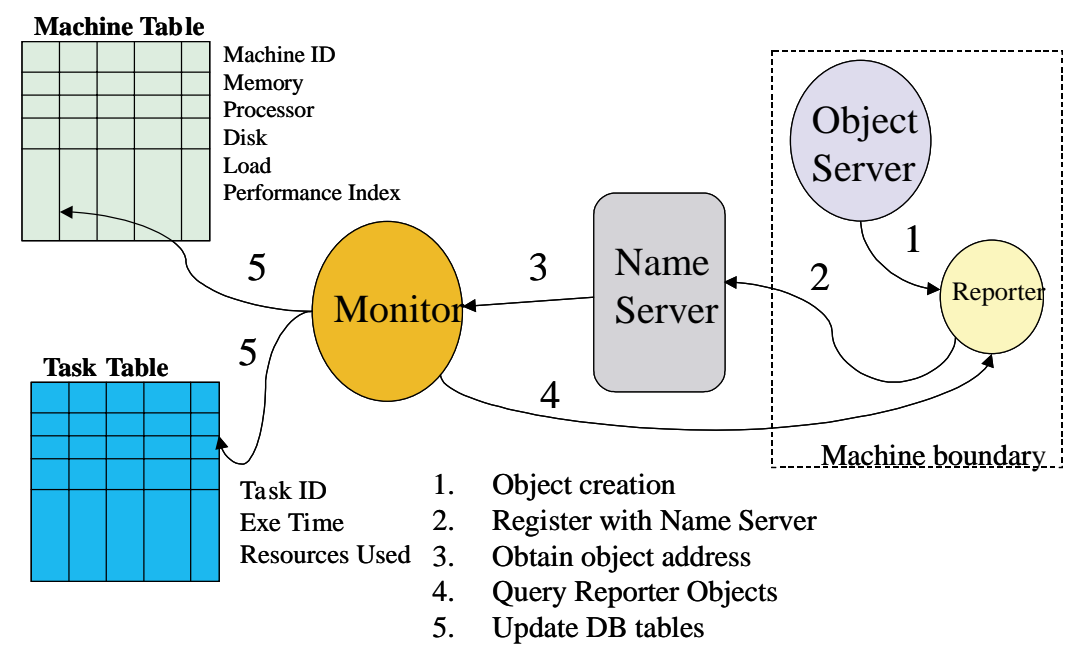

Fig. 7. Monitor interactions.

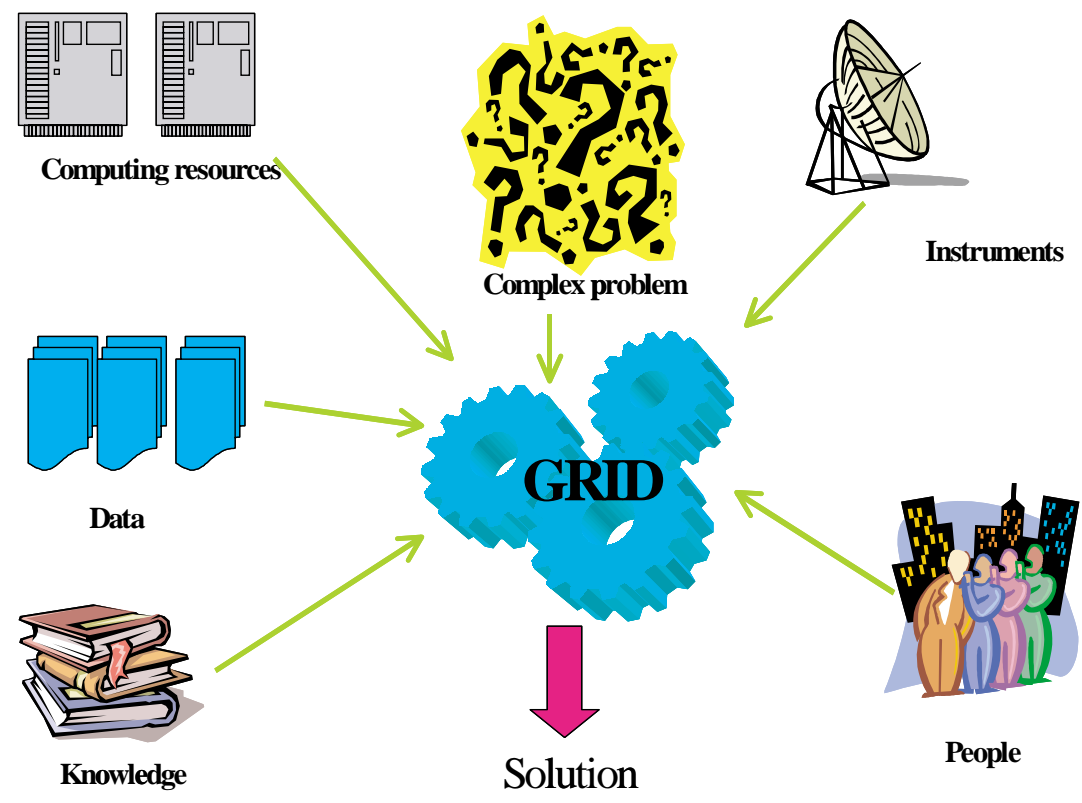

Fig. 8. Grid vision.

Science Programme', the Office of Science and Technology (OST) in the UK have allocated $£ 120 \mathrm{M}$ for the deployment of e-Science Grids spanning a wide range of application areas and the development, with industry, of the associated Grid middleware.

The Computation Grid is perhaps best envisaged as an infrastructure that integrates computing resources, data, knowledge, instruments and people. The construction of such an environment will enable sharing of computational resources, data repositories and facilities in a routine way as the Web now allows us to share information. In cartoon form, this Grid vision is depicted in Fig. 8.

There are many genuine Computer Science research challenges to be overcome before we can realize this vision. In the context of this paper, an obvious issue is the need for realistic performance estimation. Together with mechanisms for monitoring and accounting, reliable performance estimation will allow the creation of global marketplaces for Grid resources. As a starting point for a discussion of Grid performance estimation, it is worthwhile to review results from some recent 
Table 1

WAN experiment statistics

\begin{tabular}{|c|c|c|c|c|c|c|c|}
\hline \multirow[t]{2}{*}{ Partner } & \multirow{2}{*}{$\begin{array}{l}\text { CPUs } \\
\text { Nproc }\end{array}$} & \multicolumn{3}{|c|}{ Availability } & \multicolumn{3}{|c|}{ Shot statistics } \\
\hline & & In PVC & Access & Used & Failed & Successful & Total \\
\hline Southampton (PAC) & 15 & 15 & 15 & 14 & 1 & 150 & 151 \\
\hline Southampton University & 10 & 10 & 9 & 6 & 0 & 40 & 40 \\
\hline Barcelona (UPC) & 16 & 16 & 16 & 16 & 1 & 275 & 276 \\
\hline Stuttgart (RUS) & 12 & 12 & 11 & 9 & 0 & 104 & 104 \\
\hline Madrid (CASA) & 15 & 15 & 15 & 14 & 15 & 184 & 199 \\
\hline Bilbao (CEIT) & 12 & 12 & 12 & 8 & 0 & 98 & 98 \\
\hline Torino (ItalDesign) & 11 & 11 & 6 & 5 & 2 & 63 & 65 \\
\hline Torino (Blue Enginnering) & 11 & 11 & 11 & 7 & 6 & 61 & 67 \\
\hline Grand Totals & 102 & 102 & 95 & 79 & 25 & 975 & 1000 \\
\hline Total cpus installed & 102 & \multicolumn{6}{|c|}{ Elapsed Execution Time: 4:39:16 } \\
\hline Total cpus defined in PVC & 102 & \multirow{2}{*}{\multicolumn{6}{|c|}{ Approx Single CPU Time: 250 hrs }} \\
\hline Total cpus available & 95 & & & & & & \\
\hline Total cpus used in WAN & 79 & & & & & & \\
\hline
\end{tabular}

meta-computing projects.

\subsection{Meta-computing experiments}

There have been numerous meta-computing projects involving performance engineering. Here we shall restrict our discussion to several EU-funded metacomputing projects - Promenvir [35], Toolshed [32] and HPC-VAO [33]. The main application focus of these projects is engineering design optimisation by simulations. In these simulations, the parameter space of key design parameters is explored to find a set of optimal values: simulations must be performed for every new set of parameter values. The applications were drawn from a variety of engineering domains including satellite alignment analysis, surface accuracy analysis, reflector deployment, crash analysis, vibro-acoustic optimization and CFD computation.

Optimisation by simulation is computationally expensive and the user needs these simulations to execute in the shortest possible time or within a set period or resource cost. There is a clear economic incentive to achieve efficient utilisation of the available resources with as little intervention as possible. Several of these meta-computing experiments utilized Europewide computing resources. An illustration of the results of the PROMENVIR project performed by connecting up the resources of project partners across Europe is given in Table 1. Table 1 contains statistics of the resource usage obtained by running a large scale Monte Carlo simulation of satellite deployment. The program and associated data were small enough that each simulation could run on a single workstation or node: nontrivial parallelism of the application code was not required. In this simulation a thousand 'shot' (parameter set) computational experiment has been performed.
Initially all machines listed above were specified as comprising the Parallel Virtual Computer (PVC). During the actual run, however, some of them were either not available or not used due to the pre-existing high load on them. As can be seen, the experiment was very successful and utilized nearly 100 processors and resulted in a very significant improvement in exploration of the design space.

The key module of a distributed computing environment is the scheduler. This must perform task allocation to resources, run-time prediction and dynamic load-balancing. Resource management decisions must be made using platform independent application load or resource demand models for CPU, memory size, I/O traffic and disk volume. In the above mentioned metacomputing projects, such models were developed by benchmarking large industrial size codes such as NASTRAN and Sysnoise. The process of obtaining load models and using them by the scheduler is presented in Fig. 9.

The accuracy of performance predictions obtained by this technique is illustrated on the case study of a static analysis with NASTRAN [39]. It is important to stress that, as is commonly the case for commercial codes, the source code was not available for instrumentation. A series of 2D and 3D test problems were used for benchmarking on two different architectures a Distributed Memory IBM SP2 and a Shared Memory SGI Power Challenge. During benchmarking, the runtime, memory, disk traffic, disk space parameters were measured. These measurements were then used for the development of analytical performance models. At the first stage a machine independent model of the application is derived. Figure 10 illustrates that the derived CPU-load model (number of floating point operations) for SP2 and Power Challenge show a close match so 


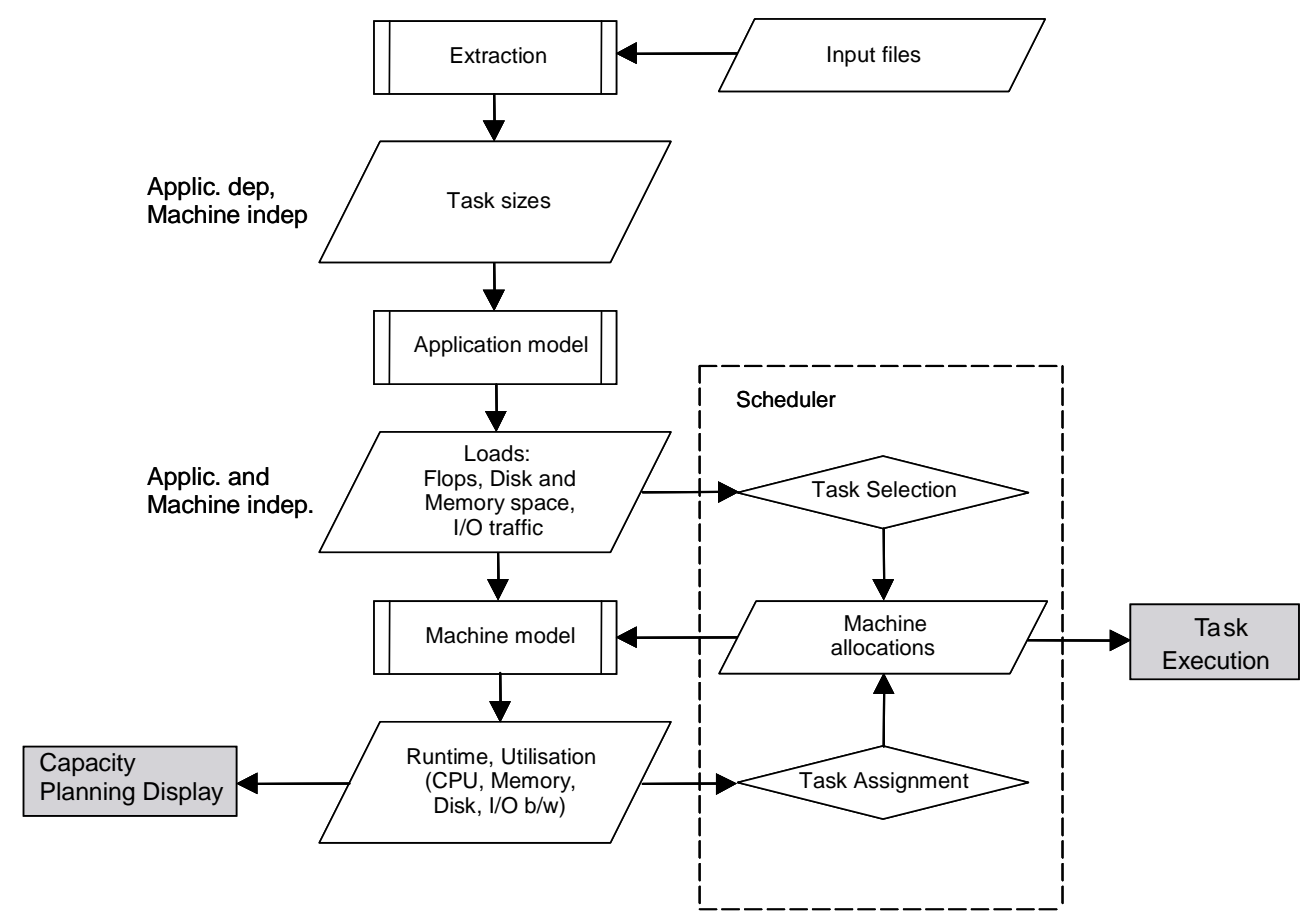

Fig. 9. Development and utilisation of platform independent load models.
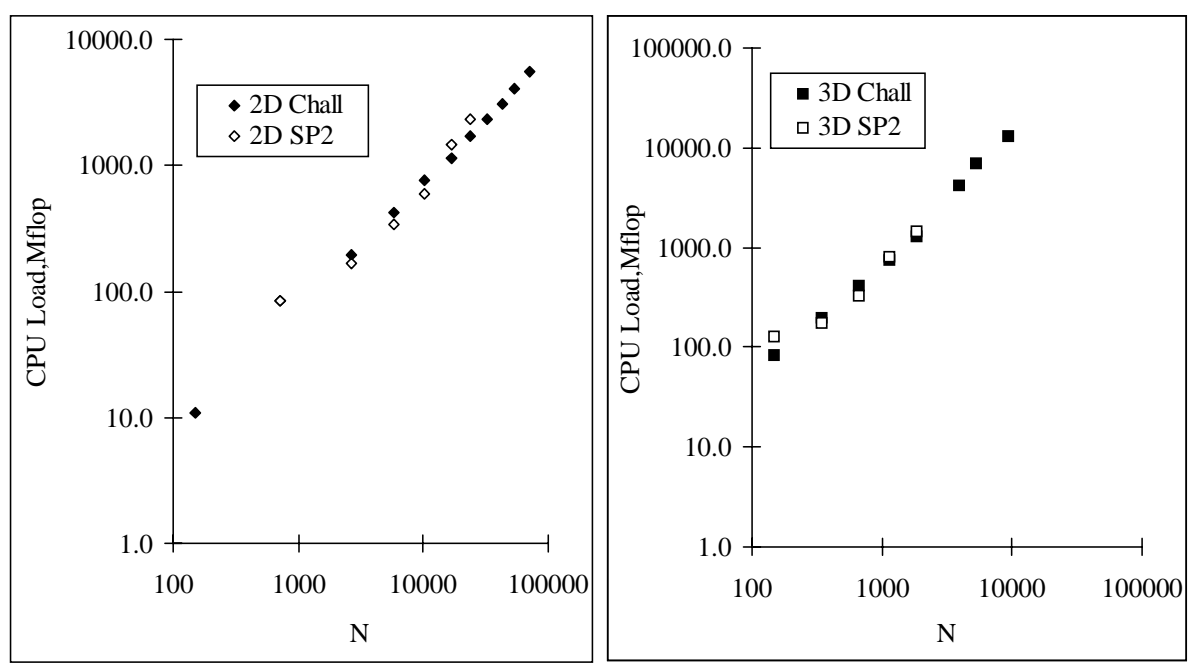

Fig. 10. Derived CPU-loads of static analysis in NASTRAN for 2D-3D problems for PowerChallenge and SP2.

that, as might be expected, the number of FPU operations in both cases is approximately the same. The derived load models are used for the development of an analytic expression that incorporates the key application parameters such as degrees of freedom, front size, number of extracted eigenvalues, etc. The accuracy of the analytical model of CPU-load for the NASTRAN static analysis code is illustrated in Fig. 11.
Similar models to the one presented in Fig. 11 have been developed for I/O load, disk volume and memory size. The advantage of this approach is that it provides simple mathematical expressions that include the key parameters governing the performance of the application. The main drawback is that the development of these models requires substantial benchmarking effort and also some knowledge of the algorithm and applica- 


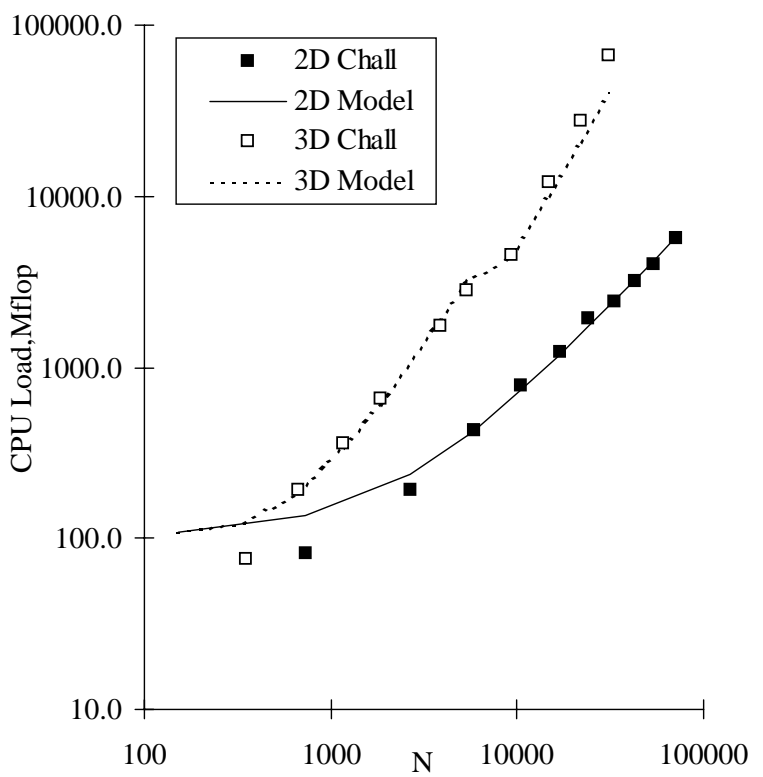

Fig. 11. CPU-load model for static analysis in NASTRAN.

tion. Nevertheless, these experiments demonstrate the level of accuracy that can be obtained in the industrially relevant environment in which the source code of the application package is unavailable.

\subsection{Performance and the Grid}

Performance estimation and forecasting will be vital ingredients of the future Grid environment as has been emphasised in several US projects such as GrADS [6], AppLeS [45] and the Network Weather Service [48]. The GrADS Project envisages a "performance contract" framework as the basis for a dynamic negotiation process between resource providers and consumers. The Network Weather Service monitors the available performance capacity of distributed resources, forecasts future performance levels using statistical forecasting models, reports monitoring data to client schedulers, applications and visual interfaces. Such a service is important for the Grid environment but needs to be scalable, portable, and secure. There are many open issues that need further investigation such as the balance between intrusiveness of sensors and accuracy of measurements, fault diagnosis and adaptive sensors.

\section{Concluding remarks}

In this paper, various techniques used for performance engineering on parallel and distributed systems have been reviewed. We conclude with two remarks:
(1) The national and international levels of investment in Grid computing make it clear that performance estimation, modelling and measurement on the Grid will assume an increasingly important role in any future computational Grid economy. Over the last decade, we have seen a shift in the software industrie towards an objectoriented, component-based software methodology. At present, although the programming interfaces and functionality of these components are exposed, there is no methodology for expressing performance trade-offs in the software development process. We therefore suggest that, in addition to specifying interfaces and functions, software methodologies need to incorporate some form of "performance metadata". Such metadata would contain information about the performance and resource requirements of software constructs and components. Only with the availability of such performance metadata will the construction of truly intelligent schedulers become possible. An internationally coordinated effort to define a common format for performance metadata seems long overdue.

(2) As we have seen, performance models range from simple algebraic models that attempt to identify a few key parameters, to complex simulation models with many parameters and involving powerful mathematical techniques such as queuing theory. However, the key to realistic performance prediction lies in understanding the interaction between the application and the computer architecture. It is also important to note that in a typical industrial application users will not have access to the source code of a software package or library routine. These requirements highlight the need for performance model abstractions that are relatively simple and easy to use yet are sufficiently accurate in their predictions to be useful as input to a scheduler or intelligent agent. Reliable performance estimation becomes even more relevant when we consider payment for services in a computational Grid economy. Users will require answers to questions such as best value for money as well as guarantees for specified turn-around times.

Finally, we have seen that there are many existing tools for performance monitoring, some of which have a non-negligible user community. When it comes to performance estimation, there are few tools and few users. Although the computer science community has 
been researching performance for a long time, we believe that such research needs to become more systematic and scientific. A common approach to performance metadata together with a methodology that allows independent verification and validation of performance results would be a good start.

\section{References}

[1] N.K. Allsopp, T.P. Cooper and P. Ftakas, Porting Legacy Engineering Applications onto Distributed NT Systems. Proceedings of the 3rd USENIX Windows NT Symposium, Seattle, Washington, USA, July 12-15, 1999.

[2] B. Alpern, L. Carter, E. Feig and T. Selker, The Uniform Memory Hierarchy Model of Computation, Algorithmica 12(2/3) (1994), 72-109.

[3] Babbage, Charles, Passages of the Life of a Philosopher, Longman, et alia, London, 1864.

[4] D. Bailey, J. Barton, T. Lasinski and H. Simon, (eds), The NAS parallelbenchmarks. Technical Report RNR-91-02, NASA Ames Research Center, Moffett Field, CA 94035, January 1991.

[5] B.P. Miller, M.D. Callaghan, J.M. Cargille, J.K. Hollingsworth, R. Bruce Irvin, K.L. Karavanic, K. Kunchithapadam and T. Newhall, The Paradyn Parallel Performance Measurement Tools, IEEE Computer 28(11) (November 1995), 37-46.

[6] F. Berman, A. Chien, K. Cooper, J. Dongarra, I. Foster, D. Gannon, L. Johnsson, K. Kennedy, C. Kesselman, D. Reed, L. Torczon and R. Wolski, The GrADS Project: Software Support for High-Level Grid Application Development, http://www.hipersoft.rice.edu/grads/publications/tr/grads project.pdf, February 15, 2000.

[7] R.G. Covington, S. Dwarkadas, J.R. Jump, J.B. Sinclair and S. Madala, Efficient Simulation of Parallel Computer Systems, International Journal in Computer Simulation 1 (1991), 3158.

[8] CRAY Research, Introducing the MPP Apprentice Tool, CRAY Manual IN-2511, 1994.

[9] D. Culler, R. Karp and D. Patterson, LogP: Towards a realistic Model for Parallel Computation, ACM SIGPLAN Notices 28(7) (1993), 1-12.

[10] A. Dunlop, Southampton Ph.D. thesis, 1997.

[11] A.C. Dusseau, D.E. Culler, K.E. Schauser and R. Martin, Fast Parallel Sorting under LogP: Experience with the CM5, IEEE Transactions on Parallel and Distributed Systems (August 1996).

[12] I. Foster and C. Kesselman, Globus: A Metacomputing Infrastructure Toolkit, International Journal of Supercomputer Applications 11(2) (1997), 115-128.

[13] I. Foster and C. Kesselman, (eds), The Grid: Blueprint for a New Computing Infrastructure, Morgan Kaufmann, 1999.

[14] T. Hey, A. Dunlop and E. Hernândez, Realistic Parallel Performance Estimation, Parallel Computing 23 (1997), 5-21.

[15] R.W. Hockney, Performance parameters and benchmarking of supercomputers, in: Computer Benchmarks, J.J. Dongarra and W. Gentzsch, eds, Elsevier Science Publishers, Holland, 1993, pp. 41-63.

[16] R.W. Hockney and M. Berry, PARKBENCH Report: Public international benchmarks for parallel computing, Scientific Programming 3(2) (1994), 101-146.
[17] W. Hoschek, J. Jaen-Martinez, A. Samar, H. Stockinger and K. Stockinger, Data Management in an International Data Grid Project, in: Proc. 1st IEEE/ACM International Workshop on Grid Computing, Springer Verlag Press, 2000.

[18] http://www.netlib.org/parkbench/.

[19] http://hpc-journals.ecs.soton.ac.uk/PEMCS/.

[20] http://www-106.ibm.com/developerworks/xml/.

[21] http://www.xml.com/.

[22] http://www.cs.purdue.edu/research/cse/gasturbn/.

[23] http://ampano.cs.utah.edu/software/.

[24] http://wwwvis.informatik.unistuttgart.de/eng/research/proj/autobench/.

[25] http://www.ipg.nasa.gov/.

[26] http://www.ncsa.uiuc.edu/About/PACI/.

[27] http://www.griphyn.org/.

[28] http://www.ppdg.net/.

[29] http://www.hoise.com/primeur/01/articles/monthly/AE-PR04-01-15.html.

[30] http://www.neesgrid.org/.

[31] http://www.eurogrid.org/.

[32] http://www.cse.clrc.ac.uk/ActivityResources/16.

[33] http://www.beasy.com/projects/hipsid/pac.html.

[34] J. Labarta, S. Girona and T. Cortes, Analyzing scheduling policies using Dimemas, Parallel Computing 23(1-2) (1997), 23-34.

[35] J. Marczyk, Principles of Simulation-Based Computer-Aided Engineering FIM Publications, Barcelona, 1999, pp. 174.

[36] W.F. McColl, BPS Programming, in Proc. DIMACS Workshop on Specification of Parallel Algorithms, Princeton, 9-11 May 1994.

[37] W.F. McColl, Truly, madly, deeply parallel, New Scientist, February 1996, pp. 36-40.

[38] F.H. McMahon, The Livermore Fortran kernels test of the numerical performance range, Performance Evaluation of $\mathrm{Su}$ percomputers (1988), 143-186.

[39] MSC/NASTRAN Quick Reference Guide, Version 70, The MacNeal-Schwendler Corporation, 1997.

[40] N. Mukherjee, G. Riley and J. Gurd, FINESSE: A Prototype Feedba-guided Performance Enhancement System, Proceedings of 8th Euromicro Workshop on Parallel and Distributed Processing, Rhodes, Greece, IEEE Computer Society Press, January 19-21, 2000, pp. 101-109.

[41] W.E. Nagel, A. Arnold, M. Weber, H.-C. Hoppe and K. Solchenbach, VAMPIR: Visualization and Analysis of MPI Resources, Supercomputer 63 12(1) (1996), 69-80.

[42] D. Pease et al., PAWS: A Performance Evaluation Tool for Parallel Computing Systems, IEEE Computer (January 1991), 18-29.

[43] S.K. Reinhardt, M.D. Hill, J.R. Larus, A.R. Lebeck, J.C. Lewis and D.A. Wood, The Wisconsin Wind Tunnel: Virtual Prototyping of Parallel Computers, in Proceedings of the 1993 ACM SIGMETRICS Conference on Measurements \& Modeling of Computer Systems, May 1993, pp. 48-60.

[44] G. Riley, M. Bull and J. Gurd, Performance Improvement through Overhead Analysis: A Case Study in Molecular Dynamics, Proceedings of the 1997 International Conference on Supercomputing, ACM Press, 1997.

[45] N. Spring and R. Wolski, Application Level Scheduling of Gene Sequence Comparison on Metacomputers, Proceedings of the 12th ACM International Conference on Supercomputing, Melbourne, Australia, July 1998.

[46] L.G. Valiant, A bridging model for parallel computation, Communications of the ACM 33(8) (1990), 103-111. 
[47] D.W. Walker, M. Li, O.F. Rana, M.S. Shields and Y. Huang, The Software Architecture of a Distributed Problem-Solving Environment Concurrency: Practice and Experience 12(15) (2001), 1455-1480.

[48] R. Wolski, Forecasting Network Performance to Support Dynamic Scheduling Using the Network Weather Service. In Proc. 6th IEEE Symp. on High Performance Distributed Computing, Portland, Oregon, 1997.
[49] Y. Zheng, N.P. Weatherill, E.A. Turner-Smith, M.I. Sotirakos, M.J. Marchant and O. Hassan, Visual Steering of Grid Generation in a Parallel Simulation User Environment, Chapter 27 in Enabling Technologies for Computational Science: Frameworks, Middleware and Environments, The Kluwer International Series in Engineering and Computer Science, (vol. 548), Kluwer Academic Publishers, Boston, 2000, pp. 339-349. 

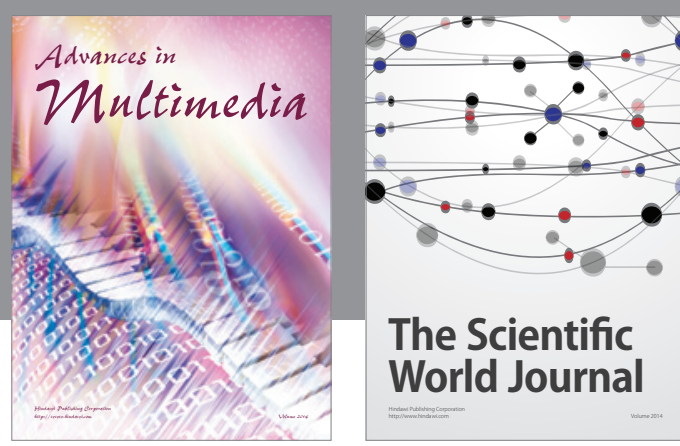

The Scientific World Journal
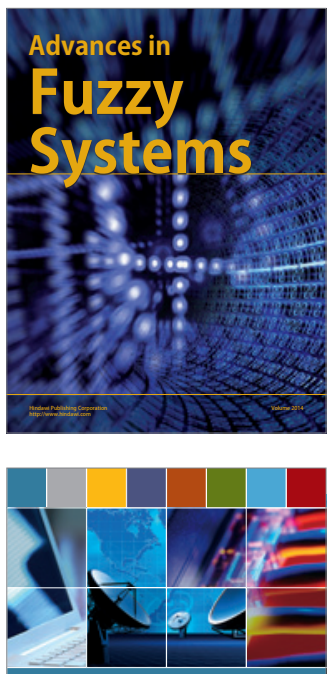

Computer Networks and Communications
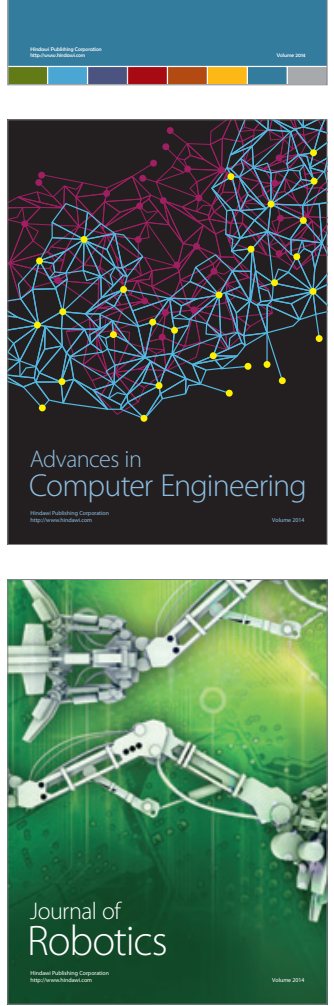
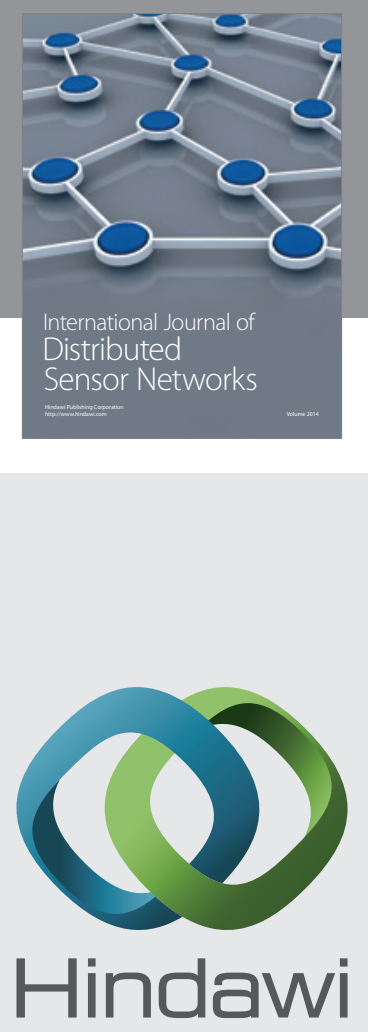

Submit your manuscripts at

http://www.hindawi.com
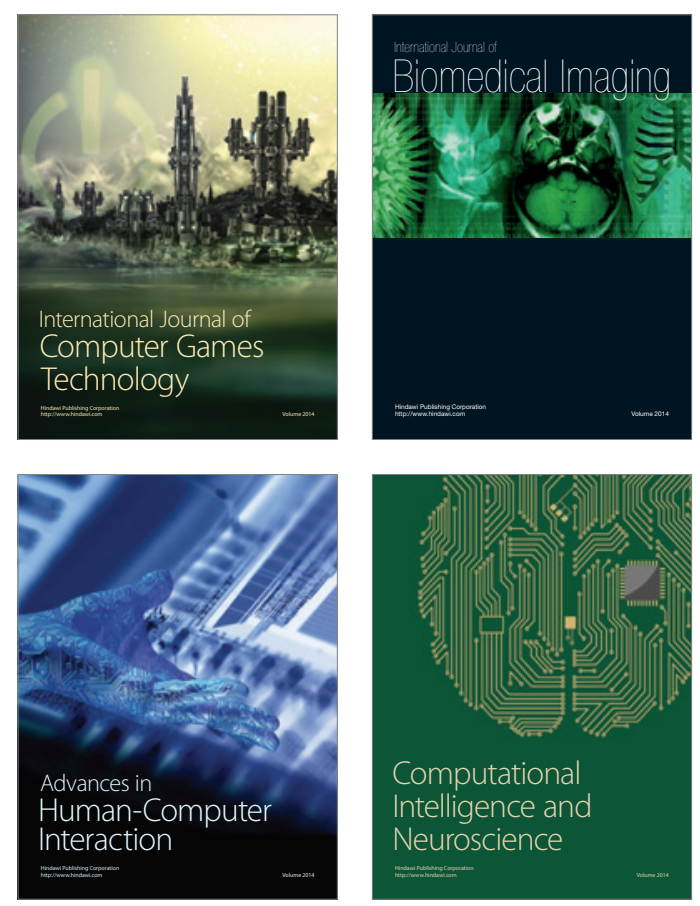
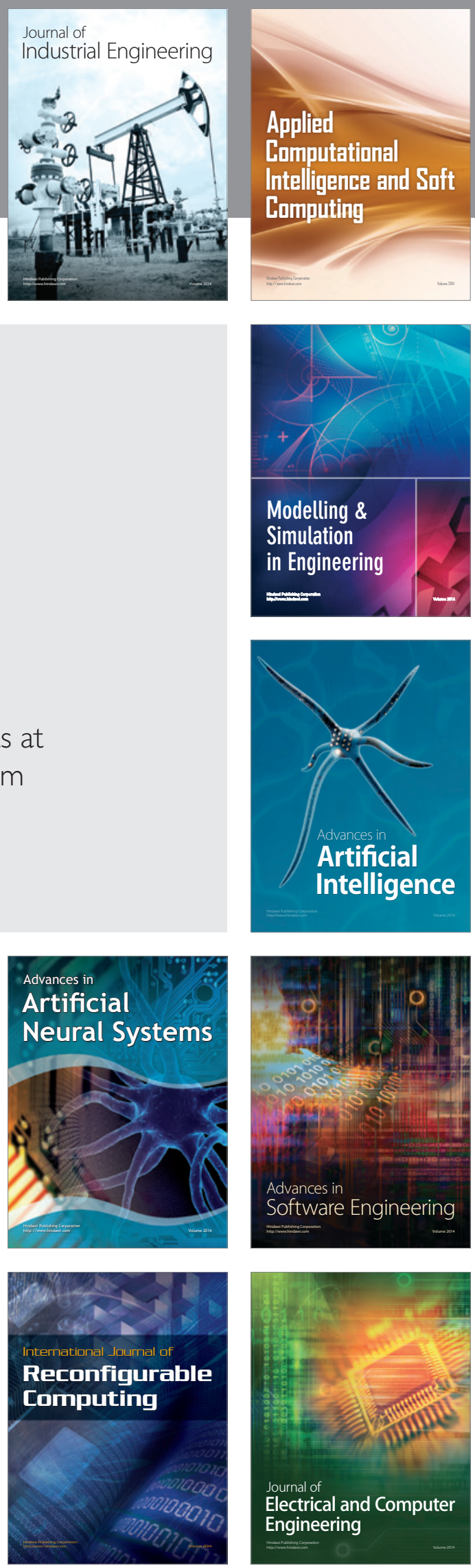